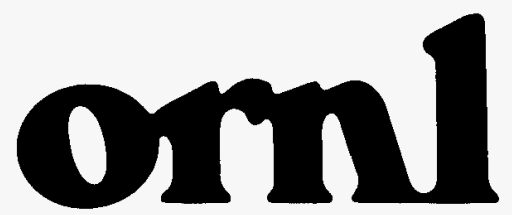

OAK RIDGE NATIONAL LABORATORY

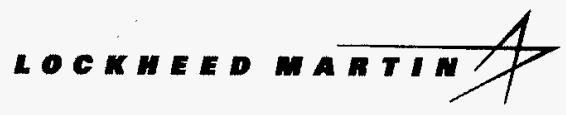

\section{RECEIVED SEP 23997 \\ $0.8 T 1$}

\title{
Integral Data Analysis for Resonance Parameters Determination
}

N. M. Larson

L. C. Leal

H. Derrien 
This repon has been reproduced directly from the best available copy:

Available to DOE and DOE contractors from the Office of Scientific and Technical Information. P.O. Box 62. Oak Ridge, TN 37831; prices available from (423) 5768401, FTS 626-8401.

Available to the public from the National Technical Information Service, U.S. Department of Commerce, 5285 Port Royal Rd., Springfield, VA 22161.

This report was prepared as an account of work sponsored by an agency of the United States Government. Neither the United States Government nor any agency thercof, nor any of their employees, makes any warranty, express or implied, or assumes any legal liability or responsibility for the accuracy, completeness, or usefulness of any information. apparatus, product, or process disclosed, or represents that its use would not infringe privately owned rights. Reference herein to any specific commercial product, process, or service by trade namc, trademark, manufacturer, or othenvise, does not necessarily constitute or imply its endorsement, recommendation, or favoring by the United Stales Government or any agency thereof. The views and opinions of authors expressed herein do not necessarily state or reflect those of the United States Government or any' agency thereof. 


\title{
Integral Data Analysis for Resonance Parameters Determination
}

\author{
N. M. Larson \\ L. C. Leal \\ H. Derrien
}

September 1997

\author{
Prepared by the \\ OAK RIDGE NATIONAL LABORATORY \\ managed by \\ LOCKHEED MARTIN ENERGY RESEARCH CORP. \\ for the \\ U.S. DEPARTMENT OF ENERGY \\ under contract DE-AC05-96OR22464
}




\section{CONTENTS}

Page

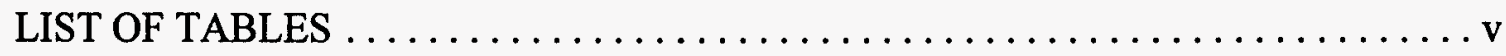

ACKNOWLEDGMENTS $\ldots \ldots \ldots \ldots \ldots \ldots \ldots \ldots \ldots \ldots \ldots \ldots$ vii

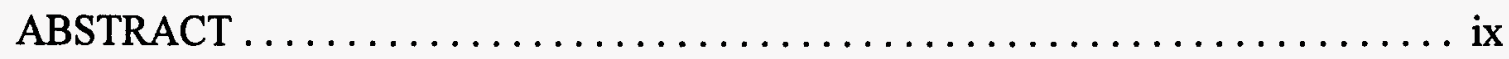

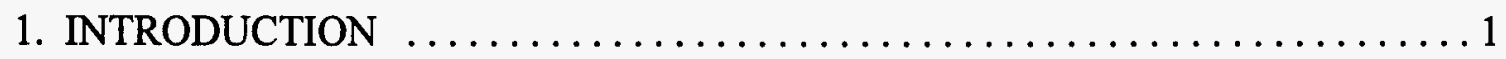

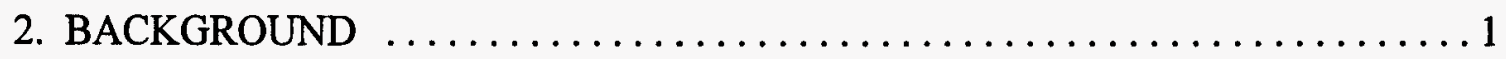

3. MATHEMATICAL DESCRIPTION OF THE INTEGRAL QUANTITIES $\ldots \ldots \ldots 2$

4. ALGEBRA FOR EVALUATING INTEGRALS $\ldots \ldots \ldots \ldots \ldots \ldots \ldots \ldots$

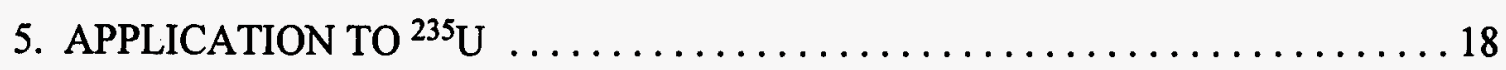

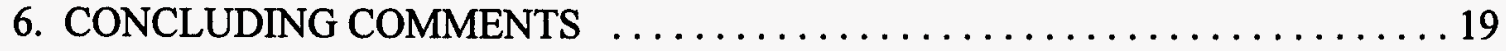

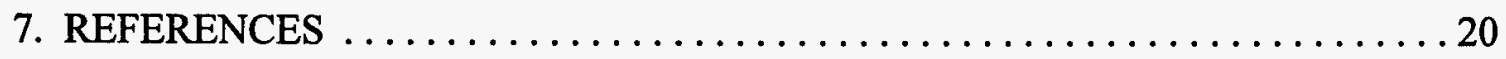

APPENDIX A. DETAILS OF THE NUMERICAL CALCULATIONS $\ldots \ldots \ldots \ldots 21$

APPENDIX B. INTEGRAL DATA FILE $\ldots \ldots \ldots \ldots \ldots \ldots \ldots \ldots \ldots \ldots \ldots \ldots \ldots \ldots \ldots$ 


\section{LIST OF TABLES}

Table

Page

1 Comparison between experimental and calculated thermal values and integral quantities. . . . . . . . 19

2 Types of integral data $\ldots \ldots \ldots \ldots \ldots \ldots 27$

3 Format of the NTG file $\ldots \ldots \ldots \ldots \ldots \ldots \ldots 28$ 


\section{ACKNOWLEDGMENTS}

This work was sponsored by Defense Programs, U.S. Department of Energy (DOE), under contract DE-AC05-960R22464 with Lockheed Martin Energy Research, Inc. The authors are particularly indebted to D. Cabrilla, DOE-Washington, for his support. 


\begin{abstract}
Neutron time-of-flight experiments have long been used to determine resonance parameters. Those resonance parameters have then been used in calculations of integral quantities such as Maxwellian averages or resonance integrals, and results of those calculations in turn have been used as a criterion for acceptability of the resonance analysis. However, the calculations were inadequate because covariances on the parameter values were not included in the calculations. In this report an effort to correct for that deficiency is documented: the R-matrix analysis code SAMMY has been modified to include integral quantities of importance, directly within the resonance parameter analysis, to determine the "best fit" to both differential (microscopic) and integral (macroscopic) data simultaneously. This modification was implemented because it is expected to have an impact on the intermediate-energy range that is important for criticality safety applications.
\end{abstract}




\section{INTRODUCTION}

Determination of integral quantities from resonance parameters until now has been performed separate from the resonance parameter analysis. First, theoretical cross sections had to be calculated from the resonance parameters and stored. From those values the integral quantities were typically generated via Simpson's rule or other integration algorithm. The integral tests were usually performed by evaluators other than the resonance parameter evaluators. Additionally, parameter uncertainties were used to determine the uncertainty on the calculation of the integral quantities. However, that determination was inherently flawed because covariances on the parameter values were not included. This deficiency has now been corrected, and it is now possible to automatically adjust parameter values (within the confines of the covariance matrix) to provide a set of parameter values and covariances that fit both the differential and the integral data. It is the purpose of this report to describe the implementation of such a procedure within the analysis code SAMMY. ${ }^{1}$

In Sect. 2 the basic procedure for analysis (fitting) of any type of data is outlined. Section 3 describes the specific functions that are currently included as part of the SAMMY code; algebraic details are given in Sect. 4. In Sect. 5 is a discussion of the application of this technique to the analysis of ${ }^{235} \mathrm{U}$. Concluding remarks are in Sect. 6. Appendix A is a description of techniques used within SAMMY for the calculation of expressions that can lead to numerical difficulties if not done carefully. Appendix B is essentially those pages for the next revision of the SAMMY manual which document how SAMMY users can access this technique.

\section{BACKGROUND}

Analysis of (microscopic or differential) neutron cross section and neutron transmission measurements involves the use of sophisticated computer models (e.g., SAMMY) to determine those values for the resonance parameters which provide the best fit of theoretical calculations to experimental measurements. The computer models incorporate the following steps:

1. Using the analyst's estimated initial values for the parameters, generate theoretical cross sections via an appropriate description of the nuclear reaction (e.g., via the ReichMoore approximation to multilevel R-matrix theory). Also, generate partial derivatives of the theoretical cross sections with respect to the resonance parameters.

2. Calculate corrections to these cross sections based on actual experimental situation. These corrections may include (but are not limited to) Doppler broadening, resolution broadening, normalization, background subtraction, and multiple-scattering 
corrections. Determine partial derivatives for any additional parameters that may be added at this stage.

3. Utilize a fitting procedure (e.g., Bayes' method, generalized least squares) to determine the "best fit" values of all the parameters.

4. Iterate steps (1-3) as needed, to adjust for nonlinearities, since the fitting procedure is inherently a linear process.

At the completion of this process, the end result is a set of values (not necessarily unique) for the resonance and data-reduction parameters, and a covariance matrix describing the accuracy to which the values are known. In essence, the square roots of the diagonal elements of the covariance matrix are the uncertainties on the parameter values, and the offdiagonal elements define their interconnectedness. Together, the values plus covariance matrix provide a complete description of the data that have been analyzed.

The values of the parameters plus the covariance matrix from analysis of one experiment can be used as input to the analysis of another experiment, and the results thus obtained provide an apt description for both measurements. This concept can of course be extended to multiple experiments.

What is new in this report is the extension of this concept into a different realm - to the analysis of nondifferential data (i.e., integral quantities).

\section{MATHEMATICAL DESCRIPTION OF THE INTEGRAL QUANTITIES}

A variety of "integral quantities" are available within SAMMY; more may be added as the need arises. In Eqs. (1-5) below, subscript $x$ is used to represent the type of cross section; that is, $x$ can be $a$ (absorption), $f$ (fission or reaction), or $c$ (capture); in Eqs. (6-9) particular types of cross sections are designated via subscripts. Though SAMMY calculates theoretical values for all 18 (for fissile nuclides) or 5 (for non-fissile) quantities, the user needs to provide only those types of data for which measurements are available.

1. Thermal cross section, ${ }^{2}$

$$
\sigma_{0 x}=\sigma_{x}\left(E_{0}\right) \quad \text { for } E_{0}=0.0253 \mathrm{eV} .
$$


2. Maxwellian average at thermal energy. ${ }^{3}$ This quantity is defined with somewhat different normalization from that used for calculation of stellar averages (see Sect. V.F of the SAMMY manual), that is, as

$$
\bar{\sigma}_{x}=\int_{E_{1}}^{E_{2}} \sigma_{x}(E) \frac{E}{E_{0}} e^{-E / E_{0}} d E / \int_{E_{1}}^{E_{2}} \frac{E}{E_{0}} e^{-E / E_{0}} d E,
$$

where $E_{1}=10^{-5} \mathrm{eV}$ and $E_{2}=3 \mathrm{eV}$.

3. Westcott's $g$-factor, ${ }^{3}$

$$
g_{x}=\frac{2}{\sqrt{\pi}} \frac{\bar{\sigma}_{x}}{\sigma_{0 x}} .
$$

4. Resonance integral, ${ }^{4}$

$$
I_{x}=\int_{E_{3}}^{E} \sigma_{x}(E) \frac{d E}{E}+X_{4 x},
$$

in which $E_{3}=0.5 \mathrm{eV}$, and $E_{4}$ and $X_{4 \mathrm{x}}$ are specified by the user. (However, in the case of $E_{4}=20 \mathrm{MeV}, X_{4 \mathrm{x}}$ is assumed to be zero.)

5. Average integral,

$$
\sigma_{x}=\int_{E_{5}}^{E_{f}} \sigma_{x}(E) d E /\left(E_{6}-E_{5}\right) .
$$

6. Watt spectrum average, ${ }^{5}$

$$
\bar{\sigma}_{W f}=\int_{E_{1}}^{E_{7}} \sigma_{f}(E) \Phi(E) d E / \int_{E_{1}}^{E_{7}} \Phi(E) d E,
$$

in which the upper limit $E_{7}=20 \mathrm{MeV}$. The Watt fission spectrum $\Phi(E)$ is given by the function

$$
\Phi(E)=e^{-E / a} \sinh (\sqrt{b E})=e^{-E / a}\left(e^{\sqrt{b E}}-e^{-\sqrt{b E}}\right) / 2 .
$$

Here $a$ and $b$ are constants to be supplied by the user. For $\mathrm{U}^{235}$ values of $a$ and $b$ are $0.988 \mathrm{MeV}$ and $2.249 \mathrm{MeV}^{-1}$, respectively. 
7. $K 1,{ }^{4}$

$$
K 1=v \sigma_{0 f} g_{f}-\sigma_{0 a} g_{a}=\left(v \bar{\sigma}_{f}-\bar{\sigma}_{a}\right) \frac{2}{\sqrt{\pi}},
$$

where $v$ is a constant provided by the user.

8. Alpha, or $\alpha^{4}$

$$
\alpha=I_{c} / I_{f}
$$

The equations shown above determine the value of the integral quantity itself; what is not shown, but is included in the computer code, is the calculation of the derivatives of the integral quantities. These are found in an identical manner, substituting the derivative for the cross section wherever it appears within the equations.

\section{ALGEBRA FOR EVALUATING INTEGRALS}

The integrations shown in Eqs. (1-9) of Section 3 can be evaluated in the following manner: First, the range of integration is divided into short regions, each region bounded by adjacent energy points from SAMMY's "auxiliary grid." This grid is chosen sufficiently dense such that the cross section between adjacent points is very nearly linear (or, for low energies, proportional to $1 / \sqrt{E}$ plus a constant). The value for the entire integral is then the sum of the integrals over the individual energy regions, with each individual region evaluated explicitly as described below. Note that the subscript $x$ on the cross sections has been deliberately omitted, for convenience's sake. In fact, the discussion below applies not only to the cross sections as stated explicitly, but also to the partial derivatives of the cross sections.

1. Thermal cross section. No integrations necessary.

2. Maxwellian

$$
\bar{\sigma}=\int_{E_{1}}^{E_{2}} \sigma(E) \frac{E}{E_{0}} e^{-E / E_{0}} d E / \int_{E_{1}}^{E_{2}} \frac{E}{E_{0}} e^{-E / E_{0}} d E .
$$

In the thermal region (low-energy region in which the cross section is monotomically decreasing), $\sigma$ may be expanded in the form 


$$
\sigma(E)=A / V+B
$$

where $V$ is the square root of $E$, and $A$ and $B$ are given by

$$
A=\frac{V_{i} V_{i+1}}{V_{i+1}-V_{i}}\left({ }_{\sigma^{i}}-\sigma_{i+1}\right)
$$

and

$$
B=\frac{\sigma_{i+1} V_{i+1}-\sigma_{i} V_{i}}{V_{i+1}-V_{i}},
$$

for $V_{i} \leq V \leq V_{i+1}$, where a subscript refers to the energy at which the quantity is evaluated; that is,

$$
V_{i}=\sqrt{E^{\prime}}{ }_{i} \text { and } \quad \sigma_{i}=\sigma\left(E^{\prime}{ }_{i}\right) \text {, }
$$

with similar expressions for $i+1$. [Primes on the energies are used to denote points within the energy grid used for evaluation of the integrands, in order to distinguish these energies from the integration limits $E_{1}$ and $E_{2}$ of Eq. (10). In the SAMMY manual ${ }^{1}$ the primed energy set is referred to as the "auxiliary energy grid."] In this case the integral from $E^{\prime}{ }_{i}$ to $E^{\prime}{ }_{i+1}$ can be written as

$$
\begin{aligned}
& \int_{E_{1}^{\prime}}^{E_{i+1}^{\prime}} \sigma(E) \frac{E}{E_{0}} e^{-E / E_{0}} d E=\int_{V_{t}}^{V_{t+1}}\left(\frac{A}{V}+B\right) \frac{V^{2}}{V_{0}^{2}} \exp \left(-\frac{V^{2}}{V_{0}^{2}}\right) 2 V d V \\
& =\frac{2 A}{V_{0}^{2}} \int_{V_{t}}^{V_{t+1}} V^{2} \exp \left(-\frac{V^{2}}{V_{0}^{2}}\right) d V+\frac{2 B}{V_{0}^{2}} \int_{V_{1}}^{V_{i+1}} V^{3} \exp \left(-\frac{V^{2}}{V_{0}^{2}}\right) d V \\
& =\frac{2 A}{V_{0}^{2}} V_{0}^{3} \int_{V_{t} / V_{0}}^{V_{i+1} / V_{0}} x^{2} e^{-x^{2}} d x+\frac{2 B}{V_{0}^{2}} V_{0}^{4} \int_{V_{t} / V_{0}}^{V_{i+1} / V_{0}} x^{3} e^{-x^{2}} d x,
\end{aligned}
$$

in which variable $x$ has been substituted for $V / V_{0}$. Inserting Eq. (12) and integrating then give 


$$
\begin{aligned}
& \int_{E^{\prime},}^{E_{1+1}^{\prime}} \sigma(E) \frac{E}{E_{0}} e^{-E / E_{0}} d E=2 V_{0} \frac{V_{i} V_{i+1}}{V_{i+1}-V_{i}}\left(\sigma_{i}-\sigma_{i+1}\right) \\
& \times\left[\frac{V_{i}}{2 V_{0}} e^{-E^{\prime}, / E_{0}}-\frac{V_{i+1}}{2 V_{0}} e^{-E^{\prime}{ }_{i+1} / E_{0}}+\frac{\sqrt{\pi}}{4}\left(\operatorname{erfc}\left(V_{i} / V_{0}\right)-\operatorname{erfc}\left(V_{i+1} / V_{0}\right)\right)\right] \\
& +2 V_{0}^{2} \frac{\sigma_{i+1} V_{i+1}-\sigma_{i} V_{i}}{V_{i+1}-V_{i}}\left[\frac{1+E^{\prime}{ }_{i} / E_{0}}{2} e^{-E^{\prime}, / E_{0}}-\frac{1+E^{\prime}{ }_{i+1} / E_{0}}{2} e^{-E_{i+1}^{\prime} / E_{0}}\right] .
\end{aligned}
$$

To simplify the notation, we replace $V_{i} / V_{0}$ by $x_{i}$ and rearrange, giving

$$
\begin{aligned}
& \int_{E^{\prime},}^{E_{1+1}^{\prime}} \sigma(E) \frac{E}{E_{0}} e^{-E / E_{0}} d E=\frac{V_{0}^{2}}{x_{i+1}-x_{i}}\left(x_{i} x_{i+1}\left(\sigma_{i}-\sigma_{i+1}\right)\right. \\
& \times\left[x_{i} e^{-x_{i}^{2}}-x_{i+1} e^{-x_{i+1}^{2}}+\frac{\sqrt{\pi}}{2}\left(\operatorname{erfc}\left(x_{i}\right)-\operatorname{erfc}\left(x_{i+1}\right)\right)\right] \\
& \left.+\left(\sigma_{i+1} x_{i+1}-\sigma_{i} x_{i}\right)\left[\left(1+x_{i}^{2}\right) e^{-x_{i}^{2}}-\left(1+x_{i+1}^{2}\right) e^{-x_{i+1}^{2}}\right]\right)
\end{aligned}
$$

or, rearranging to give coefficients of cross sections,

$$
\begin{aligned}
& \int_{E^{\prime}}^{E^{\prime}, 1+1} \sigma(E) \frac{E}{E_{0}} e^{-E / E_{0}} d E \\
& =\frac{V_{0}^{2}}{x_{i+1}-x_{i}}\left(\sigma _ { i } \left[x_{i}^{2} x_{i+1} e^{-x_{i}^{2}}-x_{i} x_{i+1}^{2} e^{-x_{i+1}^{2}}-x_{i}\left(1+x_{i}^{2}\right) e^{-x_{i}^{2}}\right.\right. \\
& \left.+x_{i}\left(1+x_{i+1}^{2}\right) e^{-x_{i+1}^{2}}+x_{i} x_{i+1} \frac{\sqrt{\pi}}{2}\left(\operatorname{erfc}\left(x_{i}\right)-\operatorname{erfc}\left(x_{i+1}\right)\right)\right] \\
& +\sigma_{i+1}\left[-x_{i}^{2} x_{i+1} e^{-x_{i}^{2}}+x_{i} x_{i+1}^{2} e^{-x_{i+1}^{2}}+x_{i+1}\left(1+x_{i}^{2}\right) e^{-x_{i}^{2}}\right. \\
& \left.\left.-x_{i+1}\left(1+x_{i+1}^{2}\right) e^{-x_{i+1}^{2}}-x_{i} x_{i+1} \frac{\sqrt{\pi}}{2}\left(\operatorname{erfc}\left(x_{i}\right)-\operatorname{erfc}\left(x_{i+1}\right)\right)\right]\right) .
\end{aligned}
$$

6 


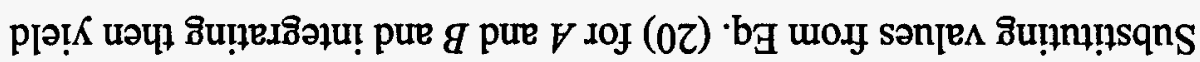

(IZ)

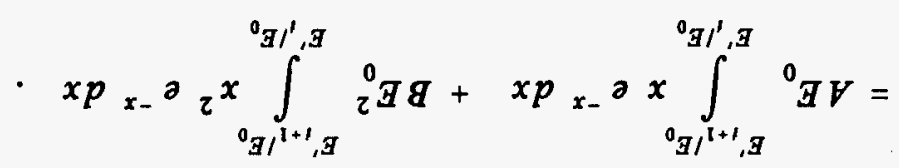

(oz)

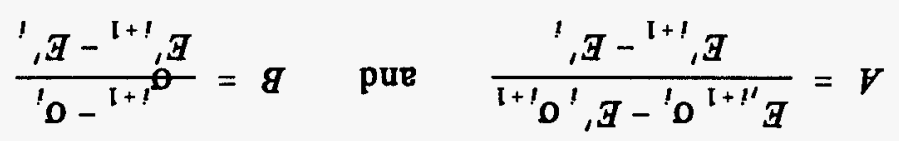

पฺ! M

(6l)

$$
\exists g+V=(\exists) 0
$$

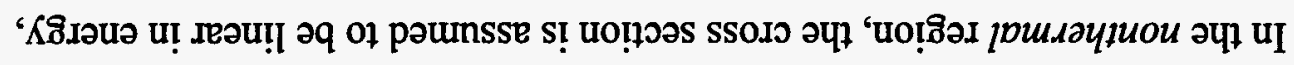

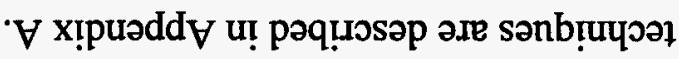

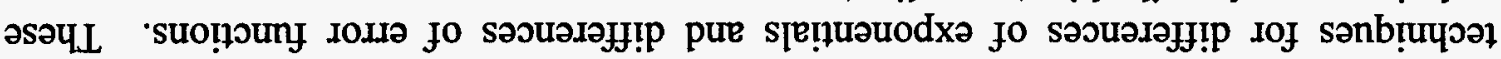

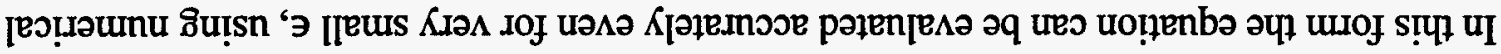

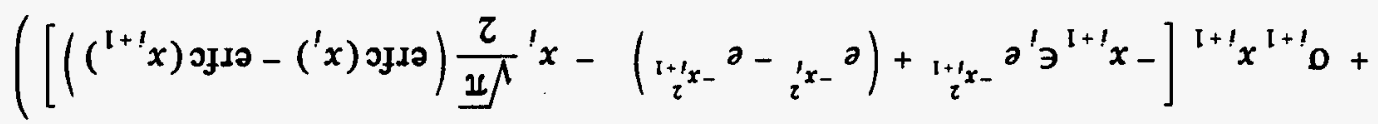

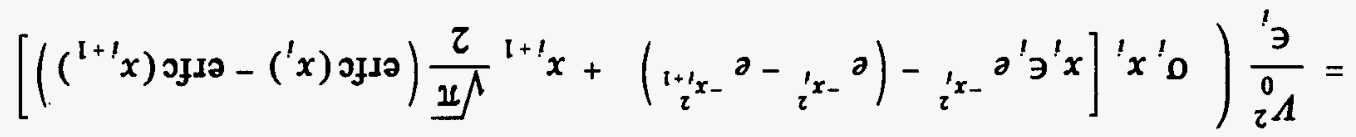

$$
\begin{aligned}
& \exists P_{0_{g / g-}} a \frac{0}{g}(g) 0 \int_{1+1, g}^{1, g}
\end{aligned}
$$

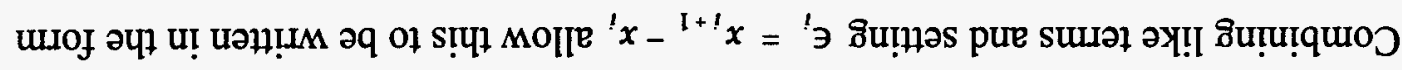




$$
\begin{aligned}
& \int_{E^{\prime}}^{E_{i+1}^{\prime}} \sigma(E) \frac{E}{E_{0}} e^{-E / E_{0}} d E \\
& =E_{0} \frac{y_{i+1} \sigma_{i}-y_{i} \sigma_{i+1}}{\delta_{i}}\left[\left(1+y_{i}\right) e^{-y_{i}}-\left(1+y_{i+1}\right) e^{-y_{i+1}}\right] \\
& +E_{0} \frac{\sigma_{i+1}-\sigma_{i}}{\delta_{i}}\left[\left(2+2 y_{i}+y_{i}^{2}\right) e^{-y_{i}}-\left(2+2 y_{i+1}+y_{i+1}^{2}\right) e^{-y_{i+1}}\right],
\end{aligned}
$$

in which we have set $y_{j}=E_{j}^{\prime} / E_{0}$ and $\delta_{i}=y_{i+1}-y_{i}$. Combining like terms and writing as coefficients of $\sigma_{i}$ and $\sigma_{i+1}$ give

$$
\begin{aligned}
\int_{E^{\prime},}^{E_{i+1}^{\prime}} \sigma(E) & \frac{E}{E_{0}} e^{-E / E_{0}} d E \\
= & E_{0} \sigma_{i}\left[\left(1+y_{i}\right) e^{-y_{i}}-\frac{\left(2+y_{i}\right) e^{-y_{1}}-\left(2+y_{i+1}\right) e^{-y_{i+1}}}{\delta_{i}}\right] \\
& -E_{0} \sigma_{i+1}\left[\left(1+y_{i+1}\right) e^{-y_{i+1}}-\frac{\left(2+y_{i}\right) e^{-y_{i}}-\left(2+y_{i+1}\right) e^{-y_{i+1}}}{\delta_{i}}\right] .
\end{aligned}
$$

As with Eq. (18), in this form the equation can be evaluated accurately even for very small $\delta$, using numerical techniques for differences of exponentials. (See Appendix A.)

The numerator of Eq. (10) is then found from Eq. (18) and (23) by summing over all energies. The denominator can be evaluated directly as

$$
\begin{aligned}
\int_{E_{1}}^{E_{2}} \frac{E}{E_{0}} e^{-E / E_{0}} d E & =E_{0} \int_{E_{1} / E_{0}}^{E_{2} / E_{0}} x e^{-x} d x \\
& =E_{0}\left[e^{-E_{1} / E_{0}}\left(1+E_{1} / E_{0}\right)-e^{-E_{2} / E_{0}}\left(1+E_{2} / E_{0}\right)\right],
\end{aligned}
$$

which, in the limit of small $E_{1}$ and large $E_{2}$, is equal to $E_{0}$.

3. Westcott's g-factor. No additional integrations needed. 
4. The resonance integral is defined as

$$
I=\int_{E_{3}}^{E_{4}} \sigma(E) \frac{d E}{E}+X_{4} .
$$

In the thermal regions the cross sections can again be approximated by Eqs. (11) and (12), giving

$$
\begin{aligned}
& \int_{E^{\prime},}^{E_{i+1}^{\prime}} \sigma(E) \frac{d E}{E}=\int_{V_{i}}^{V_{i+1}}\left(\frac{A}{V}+B\right) \frac{2 V d V}{V^{2}} \\
& \quad=2 A \int_{V_{i}}^{V_{i+1}} \frac{d V}{V^{2}}+2 B \int_{V_{i}}^{V_{i+1}} \frac{d V}{V} \\
& \quad=2 \frac{V_{i} V_{i+1}}{V_{i+1}-V_{i}}\left(\sigma_{i}-\sigma_{i+1}\right)\left(\frac{1}{V_{i}}-\frac{1}{V_{i+1}}\right)+2 \frac{\sigma_{i+1} V_{i+1}-\sigma_{i} V_{i}}{V_{i+1}-V_{i}}\left(\ln V_{i+1}-\ln V_{i}\right) \\
& \quad=\sigma_{i}\left[2-\frac{2 V_{i}}{V_{i+1}-V_{i}} \ln \frac{V_{i+1}}{V_{i}}\right]+\sigma_{i+1}\left[-2+\frac{2 V_{i+1}}{V_{i+1}-V_{i}} \ln \frac{V_{i+1}}{V_{i}}\right],
\end{aligned}
$$

in which we have again defined $V_{i}$ as $\sqrt{E_{i}{ }^{\prime}}$. Similarly, for the nonthermal regions Eqs. (19) and (20) apply, and the contribution to the resonance integral from that range is then

$$
\begin{aligned}
& \int_{E^{\prime},}^{E_{i+1}} \sigma(E) \frac{d E}{E}=\int_{E^{\prime}}^{E_{i+1}}(A+B E) \frac{d E}{E} \\
& =A \int_{E^{\prime},}^{E^{\prime}{ }_{i+1}} \frac{d E}{E}+B \int_{E^{\prime},}^{E^{\prime}{ }_{i+1}} d E \\
& =\frac{E_{i+1}^{\prime} \sigma_{i}-E^{\prime}{ }_{i} \sigma_{i+1}}{E^{\prime}{ }_{i+1}-E^{\prime}{ }_{i}}\left(\ln E^{\prime}{ }_{i+1}-\ln E^{\prime}{ }_{i}\right)+\frac{\sigma_{i+1}-\sigma_{i}}{E^{\prime}{ }_{i+1}-E^{\prime}{ }_{i}}\left(E^{\prime}{ }_{i+1}-E^{\prime}{ }_{i}\right) \\
& =\frac{E^{\prime}{ }_{i+1} \sigma_{i}-E^{\prime}{ }_{i} \sigma_{i+1}}{E^{\prime}{ }_{i+1}-E^{\prime}{ }_{i}} \ln \frac{E^{\prime}{ }_{i+1}}{E^{\prime}{ }_{i}}+\sigma_{i+1}-\sigma_{i} .
\end{aligned}
$$


This expression can be rewritten to give coefficients of the cross sections as

$$
\begin{aligned}
\int_{E^{\prime}}^{E_{i+1}^{\prime}} \sigma(E) \frac{d E}{E}=\sigma_{i}[-1 & \left.+\frac{E^{\prime}{ }_{i+1}}{E^{\prime}{ }_{i+1}-E^{\prime}{ }_{i}} \ln \frac{E^{\prime}{ }_{i+1}}{E^{\prime}{ }_{i}}\right] \\
& +\sigma_{i+1}\left[1-\frac{E^{\prime}{ }_{i}}{E^{\prime}{ }_{i+1}-E^{\prime}{ }_{i}} \ln \frac{E^{\prime}{ }_{i+1}}{E^{\prime}{ }_{i}}\right] .
\end{aligned}
$$

The resonance integral is then the sum over all energy intervals, using Eq. (26) for small energies and Eq. (28) for large. Note that the normalization on this integral is unity.

The expressions in Eqs. (26) and (28) are written in such a form as to be amenable to accurate calculation, even for the case where the two energies are relatively close to each other. Details are given in Appendix A.

Because the integration limits for the resonance integral are finite, it is possible that the integrand does not reach zero at those limits; this situation is especially likely at the lower limit, where the cross section may be exhibiting $1 / \mathrm{V}$ behavior. Therefore care must be taken to ensure that the limits are reached exactly. If $E_{i+1}^{\prime}$ represents that point from the SAMMY auxiliary energy grid directly above $E_{3}$, and if the cross section is decreasing at this energy (i.e., in thermal regions), then the contribution from that region is

$$
\begin{aligned}
\int_{E_{3}}^{E_{i+1}^{\prime}} \sigma(E) \frac{d E}{E}= & \int_{\sqrt{E_{3}}}^{V_{i+1}}\left(\frac{A}{V}+B\right) \frac{2 V d V}{V^{2}} \\
= & 2 \frac{V_{i} V_{i+1}}{V_{i+1}-V_{i}}\left(\sigma_{i}-\sigma_{i+1}\right)\left(\frac{1}{\sqrt{E_{3}}}-\frac{1}{V_{i+1}}\right)+2 \frac{\sigma_{i+1} V_{i+1}-\sigma_{i} V_{i}}{V_{i+1}-V_{i}} \ln \frac{V_{i+1}}{\sqrt{E_{3}}} \\
= & 2 \sigma_{i} \frac{V_{i}}{V_{i+1}-V_{i}}\left[\left(\frac{V_{i+1}}{\sqrt{E_{3}}}-1\right)-\ln \frac{V_{i+1}}{\sqrt{E_{3}}}\right] \\
& +2 \sigma_{i+1} \frac{V_{i}}{V_{i+1}-V_{i}}\left[-\left(\frac{V_{i+1}}{\sqrt{E_{3}}}-1\right)+\frac{V_{i+1}}{V_{i}} \ln \frac{V_{i+1}}{\sqrt{E_{3}}}\right] .
\end{aligned}
$$

If the cross sections do not exhibit "thermal" behavior (e.g., if they are increasing with energy), then the contribution from the lowest-energy region is 


$$
\begin{aligned}
\int_{E_{3}}^{E^{\prime}{ }_{i+1}} \sigma(E) \frac{d E}{E} & =\int_{E_{3}}^{E^{\prime}{ }_{i+1}}(A+B E) \frac{d E}{E} \\
& =\frac{E^{\prime}{ }_{i+1} \sigma_{i}-E^{\prime}{ }_{i} \sigma_{i+1}}{E^{\prime}{ }_{i+1}-E^{\prime}{ }_{i}} \ln \frac{E^{\prime}{ }_{i+1}}{E_{3}}+\frac{\sigma_{i+1}-\sigma_{i}}{E^{\prime}{ }_{i+1}-E^{\prime}}\left(E_{i+1}^{\prime}-E_{3}\right) \\
& =\frac{\sigma_{i}}{E^{\prime}{ }_{i+1}-E^{\prime}{ }_{i}}\left[E^{\prime}{ }_{i+1} \ln \frac{E^{\prime}{ }_{i+1}}{E_{3}}-\left({E^{\prime}}_{i+1}-E_{3}\right)\right] \\
& \quad-\frac{\sigma_{i+1}}{E^{\prime}{ }_{i+1}-E^{\prime}}{ }_{i}\left[E^{\prime}{ }_{i} \ln \frac{E^{\prime}{ }_{i+1}}{E_{3}}-\left(E^{\prime}{ }_{i+1}-E_{3}\right)\right] .
\end{aligned}
$$

Similarly, the contribution from the interval at the upper end of the integration range can be approximated as

$$
\begin{aligned}
& \int_{E^{\prime},}^{E_{4}} \sigma(E) \frac{d E}{E}=\int_{E^{\prime},}^{E_{4}}(A+B E) \frac{d E}{E} \\
& =\frac{E_{i+1}^{\prime} \sigma_{i}-E_{i}^{\prime} \sigma_{i+1}}{E_{i+1}^{\prime}-E_{i}^{\prime}} \ln \frac{E_{4}}{E^{\prime}{ }_{i}}+\frac{\sigma_{i+1}-\sigma_{i}}{E_{i+1}^{\prime}-E^{\prime}{ }_{i}}\left(E_{4}-E^{\prime}{ }_{i}\right) \\
& =\frac{\sigma_{i}}{E_{i+1}^{\prime}-E_{i}^{\prime}}\left[E_{i+1}^{\prime} \ln \frac{E_{4}}{E^{\prime}{ }_{i}}-\left(E_{4}-E_{i}^{\prime}\right)\right] \\
& -\frac{\sigma_{i+1}}{E_{i+1}^{\prime}-E^{\prime}}{ }_{i}\left[E_{i}^{\prime} \ln \frac{E_{4}{ }^{\prime}}{E}{ }_{i}-\left(E_{4}-E_{i}^{\prime}\right)\right] \text {. }
\end{aligned}
$$

5. The average integral was defined as

$$
\bar{\sigma}_{x}=\int_{E_{5}}^{E_{6}} \sigma_{f}(E) d E /\left(E_{6}-E_{5}\right)
$$

In the thermal region, contributions to this integral (numerator only) have the form 


$$
\begin{aligned}
\int_{E^{\prime}}^{E_{i}^{\prime}} \sigma(E) d E & =\int_{V_{1}}^{V_{i+1}}(A / V+B) 2 V d V \\
& =2 \int_{V_{1}}^{V_{i}}(A+B V) d V=2 A\left(V_{i+1}-V_{i}\right)+B\left(V_{i+1}^{2}-V_{i}^{2}\right) \\
& =\frac{2 V_{i+1} V_{i}\left(\sigma_{i}-\sigma_{i+1}\right)}{V_{i+1}-V_{i}}\left(V_{i+1}-V_{i}\right)+\frac{\sigma_{i+1} V_{i+1}-V_{i} \sigma_{i}}{V_{i+1}-V_{i}}\left(V_{i+1}^{2}-V_{i}^{2}\right) \\
& =\left(\sigma_{i+1} V_{i+1}+V_{i} \sigma_{i}\right)\left(V_{i+1}-V_{i}\right) .
\end{aligned}
$$

Similarly, in the nonthermal region, the cross section between adjacent grid points can be assumed to be linear in energy and given by Eqs.(17) and (18). The contribution to the average integral for two such points is then

$$
\begin{aligned}
\int_{E^{\prime}}^{E_{i}^{\prime}} \sigma(E) d E & =\int_{E^{\prime}}^{E_{1}^{\prime}}(A+B E) d E \\
& =A\left(E^{\prime}{ }_{i+1}^{1}-E^{\prime}{ }_{i}\right)+\frac{B}{2}\left(E^{\prime 2}{ }_{i+1}-E^{\prime 2}\right) \\
& =\frac{E_{i+1}^{\prime} \sigma_{i}-E^{\prime}{ }_{i} \sigma_{i+1}}{E^{\prime}{ }_{i+1}-E^{\prime}{ }_{i}}\left(E^{\prime}{ }_{i+1}-E^{\prime}{ }_{i}\right)+\frac{1}{2} \frac{\sigma_{i+1}-\sigma_{i}}{E^{\prime}{ }_{i+1}-E^{\prime}{ }_{i}}\left(E_{i+1}^{\prime 2}-E_{i}^{\prime 2}\right) \\
& =\frac{1}{2}\left(E^{\prime}{ }_{i+1}-E^{\prime}{ }_{i}\right)\left(\sigma_{i+1}+\sigma_{i}\right) .
\end{aligned}
$$

6. The Watt spectrum average is defined as

$$
\bar{\sigma}_{W f}=\int_{E_{1}}^{E_{7}} \sigma(E) \Phi(E) d E / \int_{E_{1}}^{E_{7}} \Phi(E) d E,
$$

where $\Phi(E)$ has the form

$$
\Phi(E)=e^{-E / a}\left(e^{\sqrt{b E}}-e^{-\sqrt{b E}}\right) / 2
$$


In the thermal region, the cross section has the form given by Eqs. (11) and (12). Making these substitutions and setting $E=V^{2}$ give the integral over the $i$ th energy region for the numerator of Eq. (35),

$$
\int_{E^{\prime}}^{E_{1+1}^{\prime}} \sigma(E) \Phi(E) d E=\frac{1}{2} \int_{V_{1}}^{V_{t+1}}\left(\frac{A}{V}+B\right)\left(e^{-V^{2} / a+V \sqrt{b}}-e^{-V^{2} / a-V \sqrt{b}}\right) 2 V d V .
$$

This expression can be written as the sum of two terms which differ only by signs,

$$
\int_{E^{\prime},}^{E_{1+1}^{\prime}} \sigma(E) \Phi(E) d E=I_{+}+I_{-},
$$

in which the integrals $I$ are defined as

$$
I_{ \pm}= \pm \int_{V_{i}}^{V_{t+1}}(A+B V) e^{-V^{2} / a \pm V \sqrt{b}} d V,
$$

and the obvious definitions are used for the integration limits. Setting $x=\sqrt{b E}=V \sqrt{b}$ (and making analogous definitions for the limits) gives

$$
\begin{aligned}
I_{ \pm} & = \pm \int_{x_{1}}^{x_{i+1}}(A+B x / \sqrt{b}) e^{-x^{2} / a b \pm x} d x / \sqrt{b} \\
& = \pm \int_{x_{1}}^{x_{1}+1}(A+B x / \sqrt{b}) e^{-\left(x^{2} \mp a b x+a^{2} b^{2} / 4\right) / a b+a b / 4} d x / \sqrt{b},
\end{aligned}
$$

in which the exponent was expanded by completing the square. Defining $y$ as

$$
y=\frac{x \mp \frac{a b}{2}}{\sqrt{a b}}=\sqrt{\frac{E}{a}} \mp \frac{\sqrt{a b}}{2}=\frac{V}{\sqrt{a}} \mp \frac{\sqrt{a b}}{2}
$$

(with the limit $y_{i}$ similarly expressed in terms of $x_{i}, E^{\prime}{ }_{i}$, or $V_{i}$ ) and rearranging give

$$
I_{ \pm}= \pm \sqrt{a} e^{a b / 4} \int_{y_{t}}^{y_{i+1}}(A \pm B a \sqrt{b} / 2+B \sqrt{a} y) e^{-y^{2}} d y,
$$

which can be evaluated as 


$$
\begin{gathered}
I_{ \pm}= \pm e^{a b / 4} \sqrt{a}(A \pm B a \sqrt{b} / 2) \frac{\sqrt{\pi}}{2}\left[\operatorname{erfc}\left(y_{i}\right)-\operatorname{erfc}\left(y_{i+1}\right)\right] \\
\pm e^{a b / 4} B \frac{a}{2}\left[e^{-y_{1}^{2}}-e^{-y_{i+1}^{2}}\right] .
\end{gathered}
$$

Substituting the expressions in Eq. (12) for $A$ and $B$, and defining $z$ as

$$
z_{j}=y_{j}^{2}-\frac{a b}{4}=\frac{V_{j}^{2}}{a} \mp V_{j} \sqrt{b}=\frac{E_{i j}}{a} \mp \sqrt{b E_{j}^{\prime}}
$$

give the result

$$
\begin{array}{r}
I_{ \pm}=\sigma_{i} V_{i}\left[\sqrt{a}\left( \pm V_{i+1}-a \sqrt{b} / 2\right) \frac{\sqrt{\pi}}{2} e^{a b / 4} \frac{\operatorname{erfc}\left(y_{i}\right)-\operatorname{erfc}\left(y_{i+1}\right)}{y_{i+1}-y_{i}} \frac{y_{i+1}-y_{i}}{V_{i+1}-V_{i}}\right. \\
\left.\mp \frac{a}{2} \frac{e^{-z_{i}}-e^{-z_{i+1}}}{z_{i+1}-z_{i}} \frac{z_{i+1}-z_{i}}{V_{i+1}-V_{i}}\right] \\
-\sigma_{i+1} V_{i+1}\left[\sqrt{a}\left( \pm V_{i}-a \sqrt{b} / 2\right) \frac{\sqrt{\pi}}{2} e^{a b / 4} \frac{\operatorname{erfc}\left(y_{i}\right)-\operatorname{erfc}\left(y_{i+1}\right)}{y_{i+1}-y_{i}} \frac{y_{i+1}-y_{i}}{V_{i+1}-V_{i}}\right. \\
\left.\mp \frac{a}{2} \frac{\left.e^{-z_{i}-e^{-z_{i+1}}} \frac{z_{i+1}-z_{i}}{z_{i+1}-z_{i}}\right] . V_{i+1}}{2}\right] .
\end{array}
$$

In this form the equation can be evaluated accurately even for small values of $V_{i+1}-V_{i}$. The contribution to the integrand from this energy region is then the sum of the two terms $I_{ \pm}$of Eq. (45).

In the nonthermal region, Eqs. (19) and (20) are used to approximate the value of the cross sections. Integration over the $i^{\text {th }}$ energy region gives, for the numerator of Eq. (35),

$$
\int_{E^{\prime},}^{E_{1+1}^{\prime}} \sigma(E) \Phi(E) d E=\frac{1}{2} \int_{E^{\prime}{ }_{1}}^{E_{t+1}^{\prime}}(A+B E)\left(e^{-E / a+\sqrt{b E}}-e^{-E / a-\sqrt{b E}}\right) d E
$$

Again setting $x=\sqrt{b E}$, treating the two terms of $\Phi$ separately, completing the square, and rearranging terms give 


$$
\begin{aligned}
I_{ \pm} & = \pm \frac{1}{2} \frac{2}{b} \int_{\sqrt{b E_{1}^{\prime}}}^{\sqrt{b E_{i+1}^{\prime}}}\left(A+B \frac{x^{2}}{b}\right) \exp \left[-\frac{\left(x \mp \frac{a b}{2}\right)^{2}}{a b}+\frac{a b}{4}\right] x d x \\
& = \pm \frac{1}{b} e^{a b / 4} \int_{\sqrt{b E_{t}^{\prime}}}^{\sqrt{b E_{i+1}^{\prime}}}\left(A+\frac{B}{b} x^{2}\right) x \exp \left[-\frac{\left(x \mp \frac{a b}{2}\right)^{2}}{a b}\right] d x .
\end{aligned}
$$

Setting $y$ equal to $(x \mp a b / 2) / \sqrt{a b}$ gives

$$
\begin{aligned}
& I_{ \pm}= \pm \frac{\sqrt{a b}}{b} e^{a b / 4} \int_{y_{i}}^{y_{t+1}}\left(A+B\left(a y^{2} \pm a \sqrt{a b} y+a^{2} b / 4\right)\right) \\
& \times(\sqrt{a b} y \pm a b / 2) e^{-y^{2}} d y .
\end{aligned}
$$

Eq. (48) can be rewritten as

$$
\begin{aligned}
I_{ \pm}= \pm \frac{\sqrt{a b}}{b} e^{a b / 4}[ & \pm\left(A+\frac{B a^{2} b}{4}\right) \frac{a b}{2} \int_{y_{i}}^{y_{t+1}} e^{-y^{2}} d y \\
& +\left(A \sqrt{a b}+\frac{B a^{2} b \sqrt{a b}}{4}+B \sqrt{a b a} \frac{a b}{2}\right)^{y_{1+1}} y e_{y_{i}}^{-y^{2}} d y \\
& \left.+B\left( \pm a^{2} b \pm \frac{a^{2} b}{2}\right)^{y_{i+1}} y_{y_{i}}^{2} e^{-y^{2}} d y+B a \sqrt{a b} \int_{y_{i}}^{y_{i+1}} y^{3} e^{-y^{2}} d y\right] .
\end{aligned}
$$

The third and fourth integrations in Eq. (49) may be partially evaluated as follows:

$$
\int_{y_{i}}^{y_{i+1}} y^{2} e^{-y^{2}} d y=\frac{1}{2}\left(y_{i} e^{-y_{1}^{2}}-y_{i+1} e^{-y_{i+1}^{2}}\right)+\frac{1}{2} \int_{y_{i}}^{y_{i+1}} e^{-y^{2}} d y
$$

and

$$
\int_{y_{i}}^{y_{i+1}} y^{3} e^{-y^{2}} d y=\frac{1}{2}\left(y_{i}^{2} e^{-y_{i}^{2}}-y_{i+1}^{2} e^{-y_{i+1}^{2}}\right)+\int_{y_{i}}^{y_{i+1}} y e^{-y^{2}} d y
$$


Similarly, the remaining two integrals are evaluated as

$$
\int_{y_{1}}^{y_{t+1}} e^{-y^{2}} d y=\frac{\sqrt{\pi}}{2}\left(\operatorname{erfc}\left(y_{i}\right)-\operatorname{erfc}\left(y_{i+1}\right)\right)
$$

and

$$
\int_{y_{t}}^{y_{t}+1} y^{2} e^{-y^{2}} d y=\frac{1}{2}\left(e^{-y_{t}^{2}}-e^{-y_{t+1}^{2}}\right) .
$$

With these substitutions and algebraic manipulations, the formula for $I_{ \pm}$becomes

$$
\begin{aligned}
I_{ \pm}= \pm & \frac{\sqrt{a b}}{b} e^{a b / 4}\left[ \pm\left(A+\frac{B a^{2} b}{4}+\frac{3}{2} B a\right) \frac{a b}{2} \frac{\sqrt{\pi}}{2}\left(\operatorname{erfc}\left(y_{i}\right)-\operatorname{erfc}\left(y_{i+1}\right)\right)\right. \\
& +\left(A+\frac{3}{4} B a^{2} b+B a\right) \sqrt{a b} \frac{1}{2}\left(e^{-y_{i}^{2}}-e^{-y_{i+1}^{2}}\right) \\
& \left. \pm \frac{3}{2} B a^{2} b \frac{1}{2}\left(y_{i} e^{-y_{t}^{2}}-y_{i+1} e^{-y_{i+1}^{2}}\right)+B a \sqrt{a b} \frac{1}{2}\left(y_{i}^{2} e^{-y_{i}^{2}}-y_{i+1}^{2} e^{-y_{i+1}^{2}}\right)\right] .
\end{aligned}
$$

Again the definitions of $y$ and $z$ from Eq. (41 and 44) and the definitions of $A$ and $B$ of Eq. (20) can be used to reorganize this result to give

$$
\begin{aligned}
I_{ \pm}=\sigma_{i} \frac{a^{2}}{2}\left[\left(\frac{E^{\prime}{ }_{i+1}}{a}-\frac{a b}{4}-\frac{3}{2}\right) \sqrt{a b} \frac{\sqrt{\pi}}{2} e^{a b / 4} \frac{\operatorname{erfc}\left(y_{i}\right)-\operatorname{erfc}\left(y_{i+1}\right)}{E^{\prime}{ }_{i+1}-E^{\prime}{ }_{i}}\right. \\
\pm\left(\frac{E^{\prime}{ }_{i+1}}{a}-\frac{3}{4} a b-1\right) \frac{e^{-z_{i}}-e^{-z_{i+1}}}{E^{\prime}{ }_{i+1}-E^{\prime}}{ }_{i} \\
\left.-\frac{3}{2} \sqrt{a b} \frac{y_{i} e^{-z_{i}}-y_{i+1} e^{-z_{i+1}}}{E^{\prime}{ }_{i+1}-E^{\prime}{ }_{i}} \mp \frac{y_{i}^{2} e^{-z_{i}}-y_{i+1}^{2} e^{-z_{i+1}}}{E^{\prime}{ }_{i+1}-E^{\prime}{ }_{i}}\right] \\
-\sigma_{i+1} \frac{a^{2}}{2}\left[\left(\frac{E_{i}^{\prime}}{a}-\frac{a b}{4}-\frac{3}{2}\right) \sqrt{a b} \frac{\sqrt{\pi}}{2} e^{a b / 4} \frac{\operatorname{erfc}\left(y_{i}\right)-\operatorname{erfc}\left(y_{i-1}\right)}{E^{\prime}{ }_{i+1}-E^{\prime}{ }_{i}}\right.
\end{aligned}
$$




$$
\begin{aligned}
& \pm\left(\frac{E^{\prime}{ }_{i}}{a}-\frac{3}{4} a b-1\right) \frac{e^{-z_{i}}-e^{-z_{i+1}}}{E^{\prime}{ }_{i+1}-E^{\prime}{ }_{i}} \\
& \left.-\frac{3}{2} \sqrt{a b} \frac{y_{i} e^{-z_{i}}-y_{i+1} e^{-z_{i+1}}}{E^{\prime}{ }_{i+1}-E^{\prime}{ }_{i}} \mp \frac{y_{i} e^{-z_{i}}-y_{i+1}^{2} e^{-z_{i+1}}}{E^{\prime}{ }_{i+1}-E^{\prime}{ }_{i}}\right] .
\end{aligned}
$$

In this form the expressions can again be accurately evaluated, even for small energydifference denominators. Details are given in Appendix A.

Finally, the denominator of Eq. (35) can be evaluated in the same manner as the constant terms of the cross section, to give

$$
\int_{E_{1}}^{E_{s}} \Phi(E) d E=I_{+}+I_{-},
$$

where

$$
\begin{aligned}
I_{ \pm} & =+\frac{1}{b} \int_{x_{1}}^{x_{7}} e^{-x^{2} / a b \pm x} x d x=\frac{\sqrt{a b}}{b} e^{a b / 4} \int_{y_{1}}^{y_{7}}(\sqrt{a b} y \pm a b / 2) e^{-y^{2}} d y \\
& =\frac{\sqrt{a b}}{b} e^{a b / 4}\left[\frac{\sqrt{a b}}{2}\left(e^{-y_{1}^{2}}-e^{-y_{7}^{2}}\right)+\frac{a b}{2} \frac{\sqrt{\pi}}{2}\left(\operatorname{erfc}\left(y_{1}\right)-\operatorname{erfc}\left(y_{7}\right)\right)\right] \\
& =\frac{a}{2}\left[\left(e^{-z_{1}^{2}}-e^{-z_{7}^{2}}\right)+\sqrt{a b} e^{a b / 4} \frac{\sqrt{\pi}}{2}\left(\operatorname{erfc}\left(y_{1}\right)-\operatorname{erfc}\left(y_{7}\right)\right)\right],
\end{aligned}
$$

in which the same definitions are used for $y$ and $z$ as previously, and the subscripts refer to the integration limits, that is,

$$
y_{7}=\sqrt{\frac{E_{7}}{a}} \mp \frac{\sqrt{a b}}{2}
$$

and

$$
z_{7}=\frac{E_{7}}{a} \mp \sqrt{b E_{7}}
$$

and similarly for $y_{1}$ and $z_{1}$.

7 and 8. $K 1$ and Alpha. No further integrations are needed. 


\section{APPLICATION TO ${ }^{235} \mathrm{U}$}

In the evaluations for ENDF/B-5, ${ }^{6}$ the ${ }^{235} \mathrm{U}$ cross-section representation was given by pointwise values for low energy up to $1.0 \mathrm{eV}$, rather than as a resonance parameter representation. Above $1.0 \mathrm{eV}$ the cross-section representation was given by the single-level Breit-Wigner formalism, which does not account for the long-range strong interference effects in the fission channels and therefore impacts the cross section representation at thermal energy. For ENDF/B-6 (see Ref. 7), the situation was greatly improved by using the reduced R-matrix Reich-Moore formalism for cross-section representation. In the present evaluation of Leal et al. ${ }^{8}$ it has been possible to reproduce the shape of the cross sections accurately everywhere, from a few $\mathrm{eV}$ into the $\mathrm{keV}$ energy region. Although earlier evaluations included only microscopic data in the fitting process, Hardy ${ }^{4}$ has recommended using both experimental microscopic data and experimental integral data for fitting. For the present ${ }^{235} \mathrm{U}$ evaluation both microscopic and integral data are being used.

For the resonance parameter analysis of ${ }^{235} U$, cross sections in the thermal-energy region and integral data are important for the calculation of thermal power reactors and for the interpretation of thermal critical benchmark experiments. Three integral quantities for which experimental measurements exist have therefore been included in the new analysis. These quantities are $K 1$ and the Westcott factors $g_{f}$ and $g_{a}$. The quantity $K I$, as defined in Eq. (8), is

$$
K 1=v \sigma_{0 f} g_{f}-\sigma_{0 a} g_{a} .
$$

The quantities $g_{f}$ and $g_{a}$ are given in Eq. (3),

$$
g_{x}=\frac{2}{\sqrt{\pi}} \frac{\bar{\sigma}_{x}}{\sigma_{0 x}} .
$$

In Eq. (60), $v$ is the number of neutrons per fission at thermal energy $(0.00253 \mathrm{eV}), \sigma_{0 f}$ and $\sigma_{0 a}$ are respectively fission and absorption cross sections at thermal, and $g_{f}$ and $g_{a}$ are, respectively, fission and absorption Westcott factors obtained from the cross sections averaged over a Maxwellian. Experimental values for $g_{f}$ and $g_{a}$ are given in ref. 2 and the recommended value for $K 1$ is indicated in ref. 4 .

Thermal parameters obtained in the present evaluation, first by using the microscopic experimental data only and secondly by also including the integral data, are compared to the SAMMY input experimental data in Table 1. In the fit of the microscopic data only, a strong constraint was put on the standard values, but the calculated values for the Westcott factors and for $K 1$ are too small. By including the integral data in the fitting process, excellent values are obtained for $g_{f}, g_{a}$ and $K 1$; the fission cross section at thermal $(0.00253 \mathrm{eV})$ is a little higher than the standard and the capture cross section a little lower, but both remain within the accuracy of the standard values. 
Table 1. Comparison between experimental and calculated thermal values and integral quantities.

\begin{tabular}{cccc}
\hline Quantity & $\begin{array}{c}\text { Measured, recommended, } \\
\text { and standard values }\end{array}$ & $\begin{array}{c}\text { Fit to differential } \\
\text { data }\end{array}$ & $\begin{array}{c}\text { Fit to differential plus } \\
\text { integral data }\end{array}$ \\
\hline$\sigma_{\mathrm{f}}$ & $584.25 \pm 1.11$ & 584.28 & 584.80 \\
$\sigma_{\gamma}$ & $98.96 \pm 0.74$ & 98.18 & 98.64 \\
$\sigma_{\mathrm{s}}$ & $15.46 \pm 1.06$ & 15.44 & 15.67 \\
$\mathrm{~K} 1$ & $722.7 \pm 3.90$ & 717.48 & 722.37 \\
$g_{f}$ & $0.9771 \pm 0.0008$ & 0.9743 & 0.97801 \\
$g_{\mathrm{a}}$ & $0.9790 \pm 0.0008$ & 0.9774 & 0.97995 \\
\hline
\end{tabular}

\section{CONCLUDING COMMENTS}

This report has documented a capability that permits analysis of resonance parameters by fitting to both microscopic and integral quantities. The R-matrix analysis code SAMMY has been upgraded to incorporate this capability. Application of the methodology to the evaluation of the ${ }^{235} \mathrm{U}$ cross sections was discussed; results of the analysis are in good agreement with both the microscopic and the integral results. Other integral quantities will be incorporated into SAMMY as the need arises. 


\section{REFERENCES}

1. N. M. Larson, Updated Users' Guide for SAMMY: Multilevel R-Matrix Fits to Neutron Data Using Bayes' Equations, ORNL/TM-9179/R1 (1985), /R2 (1989), /R3 (1996), Oak Ridge National Laboratory, Oak Ridge, TN.

2. A. Carlson, W. P. Poenitz, G. M. Hale, R. W. Peelle, D. C. Dodder, C. Y. Fu, and W. Mannhart, The ENDF/B-VI Neutron Cross Section Measurements Standards, NISTIR 5177, National Institute of Standards and Technology, Gaithersburg, MD, May 1993.

3. C. H. Westcott, A Study of the Accuracy of g-Factors for Room Temperature Maxwellian Spectra for U and Pu Isotopes, AECL-3255, Atomic Energy of Canada, Ltd., Chalk River (1969); see also: C. H. Westcott, "The Specification of Neutron Flux and Nuclear Cross-Sections in Reactor Calculations," J. Nuclear Energy, 2, 59-76, (1955).

4. J. Hardy, ${ }^{235}$ U Resonance Fission Integral and Alpha Based on Integral Measurements, ENDF-300, Sec. B.1, Brookhaven National Laboratory, Upton, NY (1979).

5. B. E. Wattt, "Energy Spectrum of Neutron from Thermal Fission ${ }^{235} \mathrm{U}$," Phys. Rev. 87, (1952), 1952.

6. M. R. Bhat, Summary Documentation for ${ }^{235} U$ (MAT 1395), ENDF-201, ENDF/B Summary Documentation Compiled by R. Kinsy, BNL-NCS-17541, Brookhaven National Laboratory, Upton, NY, July 1979.

7. ENDF/B-VI Summary Documentation, BNL-NCS-17541 [ENDF-201], 4th Edition, P. F. Rose, Ed. Brookhaven National Laboratory, Upton, NY, October 1991.

8. L. C. Leal, H. Derrien, N. M. Larson, and R. Q. Wright, R-Matrix Analysis of the ${ }^{235} U$ Neutron Transmission and Cross Sections in the Energy Range up to $2.25 \mathrm{keV}$, to be published as ORNL TM or in Nuc. Sci. Eng.

9. Handbook of Mathematical Table with Formulas, Graphs, and Mathematical Tables, Edited by Milton Abramowitz and Irene A. Stegun, Dover Publications, Inc., New York, NY, June 1964. 


\section{APPENDIX A. DETAILS OF THE NUMERICAL CALCULATIONS}

Evaluation of the integral quantities described in this report require numerical integration methods in which part of the integrand is known analytically (e.g., a Guassian function) and part is known on a fixed grid (e.g., the cross sections). The numerical portion must be interpolated to give values between the grid points. The interpolated function is then multiplied by the analytic function and the result integrated. This method is described in Section IV. [Alternatively, one could assume that the entire integrand exhibits linear behavior between grid points, and use, e.g., Simpson's rule to calculate the integral. In the limit of sufficiently dense mesh, the two methods should give comparable results. The method employed here, though more difficult to program initially, should be more accurate with fewer mesh points.]

One pitfall of numerical integration schemes is the necessity to evaluate functions that are ratios of two expressions, for which both denominator and numerator can be very small. (Equation (16), for example, contains this kind of function.) These functions must be evaluated by taking the limits correctly. In this appendix three such limiting cases are discussed in detail.

\section{1: Exponential}

The expression from Eq. (18) involving the differences between two exponentials,

$$
Q_{1}=\frac{e^{-x_{i+1}^{2}}-e^{-x_{i}^{2}}}{x_{i+1}-x_{i}},
$$

can be rewritten in the form

$$
Q_{1}=-e^{-x_{i}^{2}} \frac{1-e^{-\left(x_{i+1}^{2}-x_{i}^{2}\right)}}{x_{i+1}^{2}-x_{i}^{2}} \frac{x_{i+1}^{2}-x_{i}^{2}}{x_{i+1}-x_{i}}
$$

In this form the final fraction is clearly just the sum $x_{i+1}+x_{i}$. The fraction involving the exponential can be expanded using the Taylor series for exponentials,

$$
e^{-\alpha}=1-\alpha+\frac{\alpha^{2}}{2 !}-\frac{\alpha^{3}}{3 !}+\frac{\alpha^{4}}{4 !}-\ldots,
$$

so that the fraction involving the exponential becomes

$$
\frac{1-e^{-\alpha}}{\alpha}=1-\frac{\alpha}{2 !}+\frac{\alpha^{2}}{3 !}-\frac{\alpha^{3}}{4 !}+\ldots,
$$


which is a well-behaved function even for small $\alpha$.

This technique can of course be expanded to higher orders, as needed. Within SAMMY, such expressions are evaluated in subroutine $A B C E X P(X, A, B, C)$, in which $X$ is the input argument $(X=-\alpha)$, and the output variables $A, B$, and $C$ are defined as

$$
\begin{gathered}
A B C E X P(X, A, B, C)=1+X+\frac{X^{2}}{2 !}+\frac{X^{3}}{3 !}+\ldots \\
A=\frac{e^{X}-1}{X}=1+\frac{X}{2 !}+\frac{X^{2}}{3 !}+\frac{X^{3}}{4 !}+\ldots \\
B=\frac{A-1}{X}=\frac{1}{2 !}+\frac{X}{3 !}+\frac{X^{2}}{4 !}+\frac{X^{3}}{5 !}+\ldots \\
C=\frac{B-1 / 2}{X}=\frac{1}{3 !}+\frac{X}{4 !}+\frac{X^{2}}{5 !}+\frac{X^{3}}{6 !}+\ldots
\end{gathered}
$$

\section{Error functions}

A second term in Eq. (18) which requires care for dense grid points is

$$
Q_{2}=\frac{\sqrt{\pi}}{2} \frac{\operatorname{erfc}\left(x_{i}\right)-\operatorname{erfc}\left(x_{i+1}\right)}{x_{i+1}-x_{i}}
$$

A variety of numerical techniques are available for evaluation of the error function, with a range of validity appropriate to the particular technique. The technique that seems to work best for evaluating differences of error functions, in the case when the difference between the arguments is small, is to use the expansion whose first terms are given by

$$
\frac{\sqrt{\pi}}{2}[\operatorname{erfc}(X-Y)-\operatorname{erfc}(X)]=e^{-X^{2}}\left[Y+X Y^{2}+\frac{-1+2 X^{2}}{3} Y^{3}+\ldots\right] .
$$

(This result can be derived from Abromowitz and Stegun ${ }^{9}$ Eq. (7.1.5) using the binomial expansion for $(X-Y)^{2 \mathrm{n}+1}$, as shown later in this appendix.) Within SAMMY this expression is evaluated via function $A B C E R F(X, Y, A, B, C)$, in which 


$$
\begin{aligned}
& A B C E R F(X, Y, A, B, C)=Y+Y^{2} X+Y^{3} \frac{2 X^{2}-1}{3}+Y^{4} f(X, Y), \\
& A=\frac{A B C E R F}{Y}=1+Y B=1+Y X+Y^{2} \frac{2 X^{2}-1}{3}+Y^{3} f(X, Y) \\
& B=\frac{A-1}{Y}=X+Y C= \\
& C=\frac{B-X}{Y}=
\end{aligned}
$$

In these expressions the function $f$ is a polynomial in $Y$, and the coefficients of $Y^{i}$ in that polynomial $f$ are themselves polynomials in $X$.

\section{Logarithms}

For small $x$ the approximation

$$
\ln (1+x) \cong-\sum_{n=1} \frac{(-x)^{n}}{n}
$$

can be used to evaluate functions involving logarithms, such as those in Eq. (26) or (28). Within SAMMY the function ABCLOG(X,A,B,C) is used for this purpose, where

$$
\begin{aligned}
\operatorname{ABCLOG}(X, A, B, C)=\frac{1}{X} \ln (1+X) & =\sum_{n=0} \frac{(-X)^{n}}{n+1}, \\
A=\frac{1-\mathrm{ABCLOG}}{X}=\frac{1}{2}-B X & =\sum_{n=0} \frac{(-X)^{n}}{n+2}, \\
B=\frac{1 / 2-A}{X}=\frac{1}{3}-C X & =\sum_{n=0} \frac{(-X)^{n}}{n+3}, \\
C=\frac{1 / 3-B}{X} & =\sum_{n=0} \frac{(-X)^{n}}{n+4} .
\end{aligned}
$$


4. Derivation of Eq. (68)

Eq. (7.1.5) of Abromowitz and Stegun ${ }^{9}$ has the following form:

$$
\operatorname{erf}(x)=\frac{2}{\sqrt{\pi}} \sum_{n=0}^{\infty} \frac{(-1)^{n} x^{2 n+1}}{n !(2 n+1)}
$$

Since erfc $(x)=1-\operatorname{erf}(x)$, Eq. (72) can be used to express the difference

$$
\frac{\sqrt{\pi}}{2}[\operatorname{erfc}(X-Y)-\operatorname{erfc}(X)]=\sum_{n=0}^{\infty} \frac{(-1)^{n}\left[X^{2 n+1}-(X-Y)^{2 n+1}\right]}{n !(2 n+1)} .
$$

Replacing the $(X-Y)$ term by its binomial expansion gives

$$
\begin{aligned}
\frac{\sqrt{\pi}}{2}[\operatorname{erfc}(X-Y)-\operatorname{erfc}(X)]=\sum_{n=0}^{\infty} & \frac{(-1)^{n}}{n !(2 n+1)} \\
& \times\left[X^{2 n+1}-\sum_{j=0}^{2 n+1} X^{2 n+1-j} Y^{j} \frac{(2 n+1) !}{(2 n+1-j) ! j !}\right],
\end{aligned}
$$

which can be rewritten as

$$
\begin{aligned}
\frac{\sqrt{\pi}}{2}[\operatorname{erfc}(X-Y)-\operatorname{erfc}(X)]=\sum_{n=0}^{\infty} & \frac{(-1)^{n}}{n !(2 n+1)} \\
& \times \sum_{j=1}^{2 n+1} X^{2 n+1-j} Y^{j}(-1)^{j+1} \frac{(2 n+1) !}{(2 n+1-j) ! j !} .
\end{aligned}
$$

To simplify this expression, we interchange the summations and consider the coefficient of each $Y^{j}$ separately. The result is

$$
\frac{\sqrt{\pi}}{2}[\operatorname{erfc}(X-Y)-\operatorname{erfc}(X)]=e^{-X^{2}} \sum_{j=1}^{\infty} Y^{j} f_{j}(X),
$$

in which $f_{j}$ is a polynomial of degree $j-1$ in $X$. To derive the functional form for $f_{1}$ in Eq. (76), note that the $j=1$ term on the right-hand side of Eq. (75) has the form 


$$
\sum_{n=0}^{\infty} \frac{(-1)^{n}}{n !(2 n+1)} X^{2 n} Y^{1} \frac{(2 n+1) !}{1 !(2 n+1-1) !}=Y e^{-X^{2}}
$$

for $j=2$, the expression is

$$
\begin{aligned}
\sum_{n=1}^{\infty} \frac{(-1)^{n}}{n !(2 n+1)} & X^{2 n+1-2} Y^{2} \frac{(2 n+1) !(-1)^{3}}{2 !(2 n+1-2) !} \\
& =-\sum_{n=1}^{\infty} \frac{(-1)^{n}}{n !(2 n+1)} X^{2 n-1} Y^{2} \frac{(2 n+1)(2 n)}{2} \\
& =-\sum_{n=1}^{\infty} \frac{(-1)^{n}}{(n-1) !} X^{2 n-1} Y^{2} \\
& =-Y^{2} \sum_{k=0}^{\infty} \frac{(-1)^{k+1}}{k !} X^{2 k+1}=Y^{2} X e^{-X^{2}} .
\end{aligned}
$$

Continuing in this manner, one could derive $f_{j}$ for as many values of $j$ as needed to reach convergence. In summary, the first six polynomials are as follows:

$$
\begin{array}{ll}
\text { for } j=1: & f_{1}=1 \\
\text { for } j=2: & f_{2}=X \\
\text { for } j=3: & f_{3}=\left(2 X^{2}-1\right) / 3 \\
\text { for } j=4: & f_{4}=\left(2 X^{3}-3 X\right) / 6 \\
\text { for } j=5: & f_{5}=\left(4 X^{4}-12 X^{2}+3\right) / 30 \\
\text { for } j=6: & f_{6}=\left(4 X^{5}-20 X^{3}+15\right) / 90
\end{array}
$$




\section{APPENDIX B. INTEGRAL DATA FILE}

(Note: This appendix is essentially Sect. VI.I. from the SAMMY manual. ${ }^{1}$ )

When the data type is specified as "INTEGral quantities" (see Card Set 8 of the INPut file, p. 107 of the SAMMY manual), then two kinds of data files are needed. The first is an experimental differential data file, which is used (if at all) only to generate the energy-grid on which the integrals are to be calculated; the type of data and values of the cross sections in this file are ignored. The second kind of data file contains the experimental integral data itself and is designated as the "NTG" file.

In the NTG file, each type of integral data is specified by a unique five-character name, which is given in Columns 1-5 of the appropriate card. Names can be either capitals or lower case, and the ordering is arbitrary. Only those data types for which experimental measurements exist need to be specified; others can simply be omitted from this file.

Table 2 (VI.I.1 in the SAMMY manual) shows the various types of integral data available in SAMMY. Note that underscore _ in a name denotes a blank space.

Table 2. Types of integral data

\begin{tabular}{ll}
\hline Name as used in NTG file & \multicolumn{1}{c}{ Description } \\
\hline THABS, THFIS, THCAP & $\begin{array}{l}\text { Absorption, fission, or capture cross section respectively, } \\
\text { at thermal energy }(\mathrm{E}=0.0253 \mathrm{eV})\end{array}$ \\
$\begin{array}{l}\text { MXABS, MXFIS, } \\
\text { MXCAP }\end{array}$ & $\begin{array}{l}\text { Maxwellian average absorption, fission, or capture cross } \\
\text { section }\end{array}$ \\
WGABS, WGFIS, & $\begin{array}{l}\text { Westcott's g factor for absorption, fission, or capture } \\
\text { cross section }\end{array}$ \\
WGCAP & $\begin{array}{l}\text { Resonance integral for absorption, fission, or capture } \\
\text { cross section }\end{array}$ \\
RIABS, RIFIS, RICAP & $\begin{array}{l}\text { Average integral for absorption, fission, or capture cross } \\
\text { section }\end{array}$ \\
AVABS, AVFIS, AVCAP & Watt spectrum average \\
WATTS & [ $v$ (MXFIS) - (MXABS) ] $2 / \sqrt{\pi}$ \\
K1_-- & $\alpha=($ RICAP) / (RIFIS) \\
\hline
\end{tabular}


When the INPut and PARameter files specify more than one nuclide (isotope), SAMMY will calculate the integral quantities for each nuclide separately, ignoring the abundances specified in INPut and/or PARameter files. Integral data are assumed to be for a specific nuclide; it is important to note that the ordering of the nuclides must be the same in the NTG file as in the PAR file.

Correlations between experimental data are also given in the NTG file.

The name of the integral data file is given directly after the name of the differential data file, in the "interactive or batch input to SAMMY." Details for the format of the NTG file are given in Table 3 (Table VI.I.2. in the SAMMY manual).

Table 3. Format of the NTG file

\begin{tabular}{|c|c|c|c|}
\hline $\begin{array}{l}\text { Card } \\
\text { Number }\end{array}$ & Column & $\begin{array}{l}\text { Variable } \\
\text { Name }\end{array}$ & Meaning \\
\hline$\overline{1}$ & $1-70$ & TITLE & Title for the file; is printed in LPT file but never used \\
\hline 2 & $1-5$ & NUCLID & $\begin{array}{l}\text { Nuclide number (default }=1) \text {; nuclides must be in the } \\
\text { same order as in PAR file. }\end{array}$ \\
\hline \multirow[t]{5}{*}{3,4 , etc. } & $1-5$ & WHAT & Type of integral data, from list in Table VI.I.2 \\
\hline & $11-20$ & DATA & Experimental value for this data type \\
\hline & $21-30$ & UNC & Experimental uncertainty for this data type \\
\hline & $31-40$ & CONST & $\begin{array}{l}\text { For resonance integral data, CONST is the maximum } \\
\text { energy for integrand, and CONST2 is the remainder to be }\end{array}$ \\
\hline & $41-50$ & CONST2 & $\begin{array}{l}\text { added to the integrated value. } \\
\text { For average integral data, CONST is the lower limit on } \\
\text { the integral and CONST } 2 \text { the upper. } \\
\text { For K1, CONST is the value of } v \text {. } \\
\text { For Watt spectrum average, CONST }=a \text { and CONST } 2=b \text {. }\end{array}$ \\
\hline$\overline{5}$ & ? & (Blank) & \\
\hline 6 , etc. & & & Repeat cards 2-5 as many times as needed. \\
\hline 7 & & (Blank) & \\
\hline 8 & $1-5$ & WHAT & CORRElations follow \\
\hline \multirow[t]{5}{*}{9} & $1-5$ & WHAT1 & Type of integral data \\
\hline & $6-10$ & $\mathrm{NUCl}$ & Nuclide number \\
\hline & $11-15$ & WHAT2 & Type of integral data \\
\hline & $16-20$ & NUC2 & Nuclide number \\
\hline & $21-30$ & CORR & $\begin{array}{l}\text { Correlation between data of type WHAT1 for nuclide } \\
\text { number NUC1, and WHAT } 2 \text { for Nuc2 }\end{array}$ \\
\hline 10,11 , etc. & & & Repeat Card 9 as many times as needed \\
\hline 12 & & $\overline{k)}$ & \\
\hline
\end{tabular}


ORNL/TM-13495

\section{INTERNAL DISTRIBUTION}

1. B. L. Broadhead

2-7. H. Derrien

8. F. C. Difilippo

9. C. Y. Fu

10. N. M. Greene

11. K. Guber

12. J. A. Harvey

13. C. M. Hopper

14. D. T. Ingersoll

15. P. E. Koehler

16. M. A. Kuliasha

17. D. C. Larson

18-22. N. M: Larson
23-27. L. C. Leal

28. C. V. Parks

29. R. W. Roussin

30. C. H. Shappert

31. M. S. Smith

32. R. R. Spencer

33. R. M. Westfall

34. J. E. White

35. R. Q. Wright

36. RSICC

37-38. Laboratory Records for submission to OSTI

39. Laboratory Records, ORNL-RC

40. Central Research Library

\section{EXTERNAL DISTRIBUTION}

41. P. Blaise, DER/SPRC/LEPH, Batiment 230, Centre d'Etudes de CADARACHE, 13108 Saint Paul-lez-Durance, France

42. R. Block, Rensselaer Polytechnic Institute, Troy, NY 12180-3590

43. O. Bouland, DER/SPRC/LEPH, Batiment 230, Centre d'Etudes de CADARACHE, 13108 Saint Paul-lez-Durance, France

44. D. Cabrilla, U.S. Department of Energy, EM-66, Clover Leaf, Room 1199, 19901 Germantown Road, Germantown, MD 20874-1290

45. D. E. Carlson, Reactor and Plant System Branch, Division of System Research, Office of Nuclear Regulatory Research, U.S. Nuclear Regulatory Commission, MS T-10 G6, RM T-10, I7, Washington, DC 20555-0001

46. F. Corvi, Central Bureau for Nuclear Measurements, Steenweg op Retie, 2240 Geel, Belgium

47. R. L. Dintaman, U.S. Department of Energy, DP-13, Washington, DC 20585

48. C. Dunford, Bldg 197D, National Nuclear Data Center, Brookhaven National Laboratory, Upton, NY 11973

49. J. R. Felty, U.S. Department of Energy, DP-311, Washington DC 20585

50. P. Finck, Argonne National Laboratory, Reactor Analysis Division, Bldg 208, Argonne, IL 60439

51. C. M. Frankle, NIS-6, MS J562, Los Alamos National Laboratory, Los Alamos, NM 87545

52. S. C. Frankle, X-TM, MS B226, Los Alamos National Laboratory, Los Alamos, NM 87545

53. F. Froehner, Kernforschungszentrum Karlsruhe, Institut $f$. Neutronenphysik und Reacktortechnik, Postfach 336 40, D-76021 Karlsruhe, Germany

54. W. Furman, Frank Laboratory of Neutron Physics, JINR, Dubna, Russia

55. S. Ganesan, Head, Nuclear Data Section, Indira Gandhi Centra for Atomic Research, Kalpakkam 603 102, Tamilnadu, India 
56. H. Gruppelaar, Netherlands Energy Research Foundation ECN, Westerduinweg 3, P. O. Box 1, NL 1755 ZG Petten, Netherlands

57. F. Gunsing, Centre D'Etudes De Saclay, F-Saclay - 91191 GIF-SUR-YVETTE Cedex, France

58. G. M. Hale, T-2, MS B243, Los Alamos National Laboratory, Los Alamos, NM 87545

59. A. Hasagawa, Nuclear Data Center, Japan Atomic Energy Research Institute, Tokaimura, Naka-gun, Ibaraki-ken 319-11, Japan

60. R. N. Hwang, Argonne National Laboratory, Reactor Analysis Division, Bldg 208, Argonne, IL 60439

61. R. P. Jacqmin, DER/SPRC/LEPH, Batiment 230, Centre d'Etudes de CADARACHE, 13108 Saint Paul-lez-Durance, France

62. N. Janeva, Bulgarian Academy of Sciences, 72, Boul, Tzarigradsko shosse, Sofia 1784, Bulgaria

63. L. Lambros, 08 E23, U.S. Nuclear Regulatory Commission, 11555 Rockville Pike, Rockville, MD 20852-2746

64. R. Little, X-TM, MS B226, Los Alamos National Laboratory, Los Alamos, NM 87545

65. C. Lubitz, Knolls Atomic Power Laboratory, P. O. Box 1072, Schenectady, NY 12301

66. R. E. MacFarlane,T-2, MS B243, Los Alamos National Laboratory, Los Alamos, NM 87545

67. C. Mounier, CEN Saclay, DMT/SERMA/LENR, 91191 Gif Sur Yvette Cedex, France

68. M. C. Moxon, 3 Hyde Copse, Marcham, Abingdon, Oxfordshire, England

69. D. Muir, IAEA Nuclear Data Section, Wagramerstr. 5, P. O. Box 100, A-1400 Wien, Austria

70. C. W. Nilson, Office of Nuclear Regulatory Research, U.S. Nuclear Regulatory Commission, Mail Stop TWFN 9-F-33, Washington, DC 20555

71. C. Nordborg, OECD/NEA, Le Seine St-Germain 12, Boulevard Iles, 92130 Issy-lesMoulineaux, France

72. C. Raepsaet, CEN Saclay, DMT/SERMA/LEPP, 91191 Gif Sur Yvette Cedex, France

73. M. Salvatores, DRN/P, Batiment 707, C. E. CADARACHE, 13108 Saint Paul-lezDurance, France

74. E. Sartori, OECD/NEA, Le Seine St-Germain 12, Boulevard Iles, 92130 Issy-lesMoulineaux, France

75. O. A. Shcherbakov, Petersburg Nuclear Physics Institute, 188350 Gatchina, Leningrad District, Russia

76. R. Shelley, Central Bureau for Nuclear Measurements, Steenweg op Retie, 2240 Geel, Belgium

77. K. Shibata, Nuclear Data Center, Japan Atomic Energy Research Institute, Tokai-mura, Naka-gun, Ibaraki-ken 319-11, Japan

78. D. L. Smith, TD-207-DB1 16, Argonne National Laboratory, Argonne, IL 60544

79. H. Takano, Nuclear Data Center, Japan Atomic Energy Research Institute, Tokai-mura, Ibaraki-ken 319-11, Japan

80. H. Weigmann, Central Bureau for Nuclear Measurements, Steenweg op Retie, 2240 Geel, Belgium

81. C. Werner, Renssalaer Polytechnic Institute, Troy, NY 12180-3590

82. R. White, Lawrence Livermore National Laboratory, P. O. Box 808, Livermore, CA 94550

83. M. Williams, Nuclear Science Center, Louisiana State University, Baton Rouge, LA 70803 
M98054253

||||||||||||||||||||||||||||||||||||||||||||||||||||||||||||||||||

Report Number (14) ORV YTM--13495

Publ. Date (11)

Sponsor Code (18) DOE/EM, XF

UC Category (19) UC-2000, DOE/ER

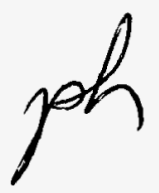

19980720095

B.T OTART ITSPEOTSDS

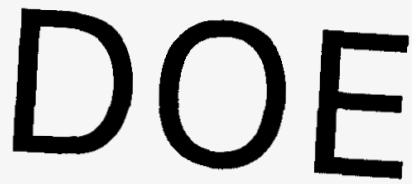

